IFIC/FTUV-9831

hep-ph/9807361

\title{
Finite dimensional systems with random external fields and Neutrino propagation in fluctuating media.
}

\author{
E. Torrente-Lujan. \\ IFIC-Dpto. Fisica Teorica. CSIC-Universitat de Valencia. \\ Dr. Moliner 50, E-46100, Burjassot, Valencia, Spain. \\ e-mail: e.torrente@cern.ch
}

\begin{abstract}
We develop the general formalism for the study of neutrino propagation in presence of stochastic media. This formalism allows the systematic derivation of evolution equations for averaged quantities as survival probabilities and higher order distribution moments. The formalism applies equally to any finite dimensional Schroedinger equation in presence of a stochastic external field. New integro-differential equations valid for finite correlated processes are obtained for the first time. For the particular case of exponentially correlated processes a second order ordinary equation is obtained. As a consequence, the Redfield equation valid for Gaussian delta-correlated noise is rederived in a simple way. The formalism, together with the quantum correlation theorem is applied to the computation of higher moments and correlation functions of practical interest in forthcoming high precision neutrino experiments. It is shown that equal and not equal time correlators follow similar differential equations.
\end{abstract}

PACS numbers: 14.60.Pq; 13.10.+q; 13.15.+g; 14.60.Gh; 96.60.Kx; 02.50.Ey; 05.40.+j; 95.30.Cq; 98.80.Cq.

Key words: neutrino, neutrino mixing, neutrino interactions, stochastic processes. 


\section{Introduction.}

Neutrino oscillations in the presence of matter and magnetic fields have been an area of intense interest for a long time. In many astrophysical situations the matter density and/or magnetic field may fluctuate around a mean value. In some scenarios it is expected that these fluctuations are very strong, for example when dealing with the magnetic fields that the neutrino encounters while propagating in the convective regions of the Sun.

The case of neutrino spin precession in a noisy magnetic field with a $\delta$-correlated Gaussian distribution was considered initially by [1] for neutrinos in vacuum. In [2], a differential equation (a Redfield equation) for the averaged matrix density was derived for the case in which the random noise was taken to be again a delta-correlated Gaussian distribution. Numerous works where approximate or numerical approaches are used to study the implications of matter random perturbations upon the SNP problem, Supernova dynamics or others are avalaible (among them: [3, 4, 5, 6, 7, 9, 10] see introductions in [2, 7] for a more extensive review).

In this work we will develop the general formalism for the study of neutrino propagation in presence of stochastic media. This formalism will allow the systematic derivation of evolution equations for averaged quantities as survival probabilities and higher order moments of them. New integro-differential equations valid for finite correlation processes are obtained for the first time. For exponentially correlated processes a second order ordinary equation is obtained as a consequence. The Redfield equation valid for Gaussian delta-correlated noise is rederived in a simple way as a particular case: it is obtained as the zero-order term of a asymptotic expansion in the inverse of the correlation length. In the context of neutrino oscillations, it is shown that the presence of matter noise induces the appearance of a effective complex part in the matter density. The presence of magnetic field noise affects however in a qualitatively different, simpler, way. The range of validity of the $\delta$-correlated model is checked in a realistic example corresponding neutrino oscillations in random solar matter. Finally the formalism, together with the quantum correlation theorem will be applied to higher moments and correlation functions of practical interest specially in forthcoming high precision neutrino experiments.

The formalism may be applied to any quantum system governed by similar Schroedinger equations: equations where a stochastic function appears multiplicatively in some part of the Hamiltonian. For simplicity of notation, in this work we will always deal with Hamiltonians which are finite dimensional operators in some Hilbert space, the equations will be obviously of the same type for general systems described by infinite dimensional Hamiltonians.

\section{The General Case.}

Let's consider a general system whose evolution is described by the linear random Schroedinger equation

$$
i \partial_{t} X=\rho(t) L(t) X ; \quad X(0)=X_{0}
$$


where: $X$ is a vector (i.e. a multiflavour wave function) or a matrix (i.e. a density matrix) of arbitrary dimension. Eq.(1) is defined in a interaction representation, any additive nonstochastic term has been solved for and absorbed in the definition of $X(t)$ and $L(t) . L(t)$ is a general linear operator. It can be an ordinary matrix if $X$ represents a wave function. If $X$ is a density matrix, $L(t)$ is a commutator:

$$
L_{A}(t) X \equiv[A(t), X]
$$

Any other linear operator is admissible: obviously it is always possible to define an enlarged vector space where the action of $L$ is represented by a matrix.

We will suppose that $\rho(t)$ in Eq.(11) is a scalar Gaussian process completely determined by its first two moments, which, without loss of generality can be taken as:

$$
\langle\rho(t)\rangle=0 ; \quad\left\langle\rho(t) \rho\left(t^{\prime}\right)\right\rangle=f\left(t, t^{\prime}\right)
$$

In other terms, $\rho(t)$ is characterized completely by the measure

$$
[d \rho] \exp -\frac{1}{2} \int_{-\infty}^{\infty} \rho(t) f^{-1}\left(t, t^{\prime}\right) \rho\left(t^{\prime}\right) d t d t^{\prime} .
$$

An important particular case is when the process is $\delta$-correlated, the correlation function is of the form:

$$
f\left(t, t^{\prime}\right)=\Omega^{2} \delta\left(t-t^{\prime}\right) .
$$

A convenient and practical way of parameterizing a correlation function with a finite correlation length is to use an exponential function:

$$
f\left(t, t^{\prime}\right)=\Omega^{2} \epsilon \exp \left(-\epsilon\left|t-t^{\prime}\right|\right)
$$

In this case the correlation length is defined as $\tau=1 / \epsilon$. The expression (5) is reobtained letting $\tau \rightarrow 0$ or $\epsilon \rightarrow \infty$ in Eq.(6).

$X(t)$, the solution to Eq.(11) is a stochastic function. The objective of this work is to obtain in a systematic way equations for its ensemble average and higher moments. Let us remark that for us Eq.(1) is purely phenomenological, we suppose that is the result of a more complete microscopic analysis which can account for the randomness of $\rho(t)$ (see for example []]).

To obtain a differential equation for the average of $X$ we make use of the following well known property: For any Gaussian process $\rho(t)$ characterized by a $\delta$-correlation function (6) and any functional $F[\rho]$ we have the following relation ([11]):

$$
\langle F[\rho] \rho(t)\rangle=\Omega^{2}\left\langle\frac{\delta F[\rho]}{\delta \rho(t)}\right\rangle .
$$

For a Gaussian process with arbitrary correlation function (Eq.(4)) we have the general relation:

$$
\langle F[\rho] \rho(t)\rangle=\int d \tau\langle\rho(t) \rho(\tau)\rangle\left\langle\frac{\delta F[\rho]}{\delta \rho(\tau)}\right\rangle
$$


Let's consider now the evolution operator $U$ for a particular realization of Eq.(1); by definition:

$$
X(t)=U\left(t, t_{0}\right) X_{0}
$$

The operator $U\left(t, t_{0}\right)$ is a functional of $\rho$ and it has the following formal expression in terms of a time ordered exponential:

$$
U\left(t, t_{0}\right)=T \exp -i \int_{t 0}^{t} \rho(\tau) L(\tau) d \tau
$$

The functional derivative of $U$ with respect to $\rho(t)$ can be computed by direct methods differentiating term by term the series expansion for Eq.(10). The result is:

$$
\frac{\delta U\left(t, t_{0}\right)}{\delta \rho(\tau)}=-i L(\tau) U\left(\tau, t_{0}\right), \quad t_{0}<\tau<t
$$

In order to obtain a differential equation for $\langle X\rangle$ we observe that

$$
i\left\langle\partial_{t} X\right\rangle=i \partial_{t}\langle X\rangle=L(t)\langle\rho(t) X\rangle .
$$

Combining together Eq.(8.9.11) we obtain easily that the evolution equation for the ensemble average is in the general case a integro-differential equation given by:

$$
i \partial_{t}\langle X(t)\rangle=-i \int_{0}^{t} d t^{\prime} f\left(t, t^{\prime}\right) L(t) L\left(t^{\prime}\right)\left\langle X\left(t^{\prime}\right)\right\rangle, \quad\langle X(0)\rangle=X_{0}
$$

This equation, which is exact and of very general validity, is the equation we were looking for and one of the main result of the present work. Note that it has been obtained previously integro-differential equations, valid for particular cases or in particular limits, using heuristic ad-hoc arguments (for example in [10]). The derivation of Eq. 13) done here is the rigorous justification for such approaches.

\section{The particular $\delta$-correlated case.}

For the particular case where the correlation function is of exponential type, a second order ordinary differential equation can be obtained as we will see below. In the other hand, for the simpler $\delta$-correlated case the evolution equation (13) becomes the purely differential equation:

$$
i \partial_{t}\langle X(t)\rangle=-i \Omega^{2} L^{2}(t)\langle X(t)\rangle .
$$

Taking $L$ as a commutator, this last equation coincides with the Redfield equation derived by [2]. Note that the effective "Hamiltonian" appearing in the second part of Eq.(14) is not anymore hermitic ( an example of a fluctuation-dissipation effect).

In practical cases of interest for the neutrino oscillation problem, the original equation for the density matrix is of the slightly simpler form:

$$
i \partial_{t} X=\left[H_{0}(t)+\rho(t) g(t) H_{1}, X\right] ; \quad X(0)=X_{0}
$$


where we have written explicitly the commutator. $\rho(t)$ is a stochastic function as before and $g(t)$ an arbitrary scalar function. $H_{0}, H_{1}$ are Hamiltonian matrices, the former contains the average part of $\rho(t): H_{0} \equiv H_{0}^{\prime}(t)+\rho_{0}(t) H_{1}$, with $\rho_{0}=\langle\rho\rangle$. The latter is supposed to be time independent.

For the problem described by Eq. (15) the corresponding Redfield equation for the averaged density matrix is of the form

$$
\begin{aligned}
i \partial_{t}\langle X\rangle & =\left[H_{0}(t),\langle X\rangle\right]-i \Omega^{2} g^{2}(t)\left[H_{1},\left[H_{1},\langle X\rangle\right]\right] . \\
& \equiv H_{0}^{-}\langle X\rangle-\langle X\rangle H_{0}^{+}+2 i \Omega^{2} g^{2}(t) H_{1}\langle X\rangle H_{1}
\end{aligned}
$$

where in the last line the following effective Hamiltonians were defined:

$$
H_{0}^{ \pm}=H_{0} \pm i g^{2}(t) H_{1}^{2} .
$$

The solution of what is called the "coherent" part of Eq.(17) is accomplished by defining the average evolution operator:

$$
\left\langle U^{ \pm}\right\rangle=T \exp \int d \tau H_{0}^{ \pm}(\tau) ; \quad\left\langle U^{-}\right\rangle=\left\langle U^{+}\right\rangle^{\dagger} .
$$

The coherent part of the density matrix is then:

$$
\langle X\rangle_{c o h}=\left\langle U^{-}\right\rangle X_{0}\left\langle U^{+}\right\rangle^{\dagger}
$$

Defining a new "coherent" interaction representation by the relations:

$$
H_{P}=\left\langle U^{-}\right\rangle^{-1} H_{1}\left\langle U^{-}\right\rangle, \quad H_{Q}=\left\langle U^{-}\right\rangle H_{1}\left\langle U^{-}\right\rangle^{-1}, \quad\langle X\rangle_{I}=\left\langle U^{-}\right\rangle\langle X\rangle_{c o h}\left\langle U^{-}\right\rangle^{-1},
$$

the resolution of the original equation is equivalent to the resolution of the following one:

$$
i \partial_{t}\langle X\rangle_{I}=2 i g^{2}(t) \Omega^{2} H_{P}\langle X\rangle_{I} H_{Q} .
$$

There are some important particular practical cases where Eq.(14) can be solved or simplified considerably taking into account the algebraic properties of a specific $L$ (in what follows $k(t)$ is always a scalar function):

A. Let us suppose that $L$ is such that $L^{2}(t)=k(t) L(t)$. This case appears in the computation of the average wave function with matter density noise. Eq.(14) reduces to:

$$
i \partial_{t}\langle X\rangle=-i \Omega^{2} k(t) L(t)\langle X\rangle .
$$

The averaged equation is similar to the original one, the non-random part of the density is "renormalized" acquiring an imaginary term:

$$
\rho \rightarrow \rho_{0}-i \Omega^{2} k \text {. }
$$

This is the density which will appear in the coherent effective Hamiltonians $H_{0}^{ \pm}$. This case was discussed already in [5]. Enquist and Semikoz ([3]) saw numerically that the net 
effect in the averaged survival probability of the same system is a precession reduction, similar to the effect of a larger constant matter density. It can be argued (Eqs. (19) through (21)) that the complex renormalization defined before appears also, but this time approximately, if we compute average probabilities. Under this perspective the reduction in neutrino precession can be explained as a smoothening of the MSW resonance induced by the imaginary term. It is expected from here that $\delta$-correlated matter noise have importance only if applied over a MSW resonance region.

B. The case where $L^{2}(t)=k(t) I$ with $I$ the identity matrix appears in the computation of the averaged neutrino wave function under noisy magnetic spin-flavour precession. The resulting equation can be integrated trivially (to be compared with the previous case):

$$
\langle X(t)\rangle=\exp -\Omega^{2} \int_{0}^{t} d \tau k(\tau) X_{0} .
$$

The average wave oscillation is damped by a factor equivalent to the one first calculated by Nicolaidis ([1]). This damping manifest itself also when computing the average density matrix from Eq.(21). From these difference of behavior with respect case (A) it is expected that magnetic field noise can affect even if applied far from any resonance region.

C. The case where $L^{4}(t)=-k(t) L^{2}(t)$ appears in the computation of full average density matrix with both, matter density or magnetic noise. We can obtain in this case the "conservation law":

$$
\left(1-\Omega^{2} k(t)\right) L^{2}(t) \partial_{t}\langle X(t)\rangle=0 .
$$

$L^{2}(t)$ is not invertible because the operator $L(t)$ has a zero eigenvalue. The previous expression has proved to be of practical importance in some concrete numerical applications $(12,13,14])$.

\section{An Asymptotic Expansion for Exponentially correlated systems.}

We will see now how the Eq.(14) can be obtained as a limiting particular case when the correlation length tends to zero. For this purpose we use an exponential correlation function as Eq.(6), the integro-differential evolution equation becomes in this case:

$$
i \partial_{t}\langle X(t)\rangle=-i \Omega^{2} \epsilon \exp (-\epsilon t) \int_{0}^{t} d t^{\prime} \exp (\epsilon \tau) L(t) L\left(t^{\prime}\right)\left\langle X\left(t^{\prime}\right)\right\rangle
$$

Let us compute the asymptotic expansion of the second term of Eq.(22) valid for $\epsilon$ large, the following expansion is valid for any function $g(t)$

$$
h(\epsilon) \equiv \epsilon \exp (-\epsilon t) \int_{0}^{t} d \tau \exp (\epsilon \tau) g(\tau) \sim g(t)-\frac{g^{\prime}(t)}{\epsilon}+\frac{g^{\prime \prime}(t)}{\epsilon^{2}}+\ldots
$$

Inserting this expression in Eq.(22), we obtain the following expansion in powers of $\epsilon$ :

$$
i \partial_{t}\langle X\rangle=-i \Omega^{2} L^{2}(t)\langle X\rangle+i \frac{\Omega^{2}}{\epsilon} L(t) \partial_{t}(L(t)\langle X\rangle)+o\left(\frac{1}{\epsilon^{2}}\right)
$$


To leading order in $1 / \epsilon$, we recover the expression corresponding to the $\delta$-correlated case. At next-to-leading order we get finite-correlation correction terms:

$$
i \partial_{t}\langle X\rangle=-i \Omega^{2} L^{2}(t)\langle X\rangle+i \frac{\Omega^{2}}{\epsilon} L(t) L^{\prime}(t)\langle X\rangle+i \frac{\Omega^{2}}{\epsilon} L^{2}(t) \partial_{t}\langle X\rangle
$$

or equivalently

$$
\left(1-\frac{\Omega^{2}}{\epsilon} L^{2}(t)\right) \partial_{t}\langle X\rangle=\left(-\Omega^{2} L^{2}(t)+\frac{\Omega^{2}}{\epsilon} L(t) L^{\prime}(t)\right)\langle X\rangle
$$

We finally get the following differential equation valid to order $1 / \epsilon$, making the supposition that the operator which multiplies the first term is invertible,

$$
\partial_{t}\langle X\rangle=\left(-\Omega^{2} L^{2}(t)+\frac{\Omega^{2}}{\epsilon} L(t) L^{\prime}(t)+\frac{\Omega^{4}}{\epsilon} L^{4}(t)\right)\langle X\rangle
$$

This equation can be used for finite, but relatively large, correlation lengths. We see that, up to this degree of approximation, not only the ratio level of noise to correlation length $\left(\Omega^{2} / \epsilon\right)$ is important. We have different regimes according to the value of $\Omega^{2}$. For low noise amplitude $\left(\Omega^{2}<<1\right)$ the first term will be more important. For strong noise $\left(\Omega^{2}>>1\right)$ the second one, proportional in this case to $L^{4}$, will dominate.

We have pointed out previously that in the practical cases of interest for neutrino oscillation problems the relation $L^{4}(t)=k(t) L^{2}(t)$ holds, with $k(t)$ positive. If in addition the term $L^{\prime}(t)$ can be neglected, as indeed happens in some occasions, Eq.(27) becomes:

$$
\partial_{t}\langle X\rangle=-\Omega^{2}\left(1-\frac{\Omega^{2}}{\epsilon} k(t)\right) L^{2}(t)\langle X\rangle
$$

In this case and within this level of approximation the net effect of the presence of a finite correlation length is visible: it amounts to a rescaling and consequent reduction of the noise parameter $\Omega^{2}$.

\section{An Exact Differential Equation for Exponentially Corre- lated Systems.}

In contrast with the approximate approach used in the previous section, we can derive in fact a simple, ordinary second order differential equation for the case of exponential correlation. Let's suppose that the original equation is of the same decomposable type as the one appearing in Eq.(15) but let's include other cases using the general notation:

$$
i \partial_{t} X=\left(L_{0}(t)+\rho(t) g(t) L_{1}\right) X ; \quad X(0)=X_{0}
$$

with $L_{0}, L_{1}$ general linear operators as before, the latter time-independent. In this case Eq.(13) is of the form

$$
i \partial_{t}\langle X\rangle=L_{0}(t)\langle X\rangle-i \Omega^{2} \epsilon L_{1}^{2} e^{-\epsilon t} g(t) \int_{0}^{t} d t^{\prime} g\left(t^{\prime}\right) e^{\epsilon t^{\prime}}\left\langle X\left(t^{\prime}\right)\right\rangle .
$$


Differentiating the equation once and after some simple algebra we obtain the following ordinary second order differential equation:

$$
\begin{aligned}
\left(\partial_{t}-\lambda(t)\right)\left[i \partial_{t}-L_{0}(t)\right]\langle X\rangle & =-i \Omega^{2} \epsilon g^{2}(t) L_{1}^{2}\langle X\rangle \\
i\left(\partial_{t}\langle X\rangle\right)_{0} & =L(0)\langle X\rangle_{0}, \quad\langle X\rangle_{0}=X_{0}
\end{aligned}
$$

with $\lambda(t) \equiv-\epsilon+g^{\prime}(t) / g(t)$. Let's remark that this equation is exact, unless the approximations we have seen in the previous section.

As illustration and for the sake of comparation, we have solved Eqs.(31) and (14) for the case of two-flavour ultrarelativistic neutrino oscillations in presence of solar matter with a random perturbation. The function $g(t)$ is in this case proportional to the solar electron density profile and can be approximated rather well by an exponential function (see notation and details in [2] for example). The results of the numerical calculations are shown in Figs.(1) (A) (Eq.(14)) and (B) (Eq.(31)). In plot (A) we observe the typical structure of the solar MSW resonance as a function of the neutrino energy and how this resonance is modified by the presence of the $\delta$ correlated chaotic field. The broad range of validity of Eq.(14), considered as an approximation to the more realistic Eq.(31) is apparent in plot $(\mathrm{B})$. We observe there how the survival probability depends on $\epsilon$. The neutrino energy is fixed in this case to be at the center of the resonance region and corresponds approximately to a neutrino oscillation length of $3.110^{3} \mathrm{Km}, 410^{-3} \mathrm{R}_{\odot}$ or $810^{-3}$ times the distance over the which the noise is acting. For any fixed value of the quantity $\Omega^{2}$, the limit of small $\epsilon$ (large $\mathrm{L} / \mathrm{d}$ in the figure) coincide with the noise-less case. After a relatively narrow transition region, for larger, but in fact not so large, $\epsilon$ ( $L \approx d)$ the probability tends to a constant; the one which is obtained from Eq.(14). The conclusion is that, at least for the example in consideration, the $\delta$-correlated noise is a good model even for "not so small" correlation lengths. I

\section{Higher Order Moments and the Quantum Regression The- orem.}

Second order moments, expressions of the type $\left\langle X_{i} X_{j}\right\rangle$, or in general, moments of any order, can be computed also using equations similar to Eq.13). The straightforward procedure is to define products $X_{i j . . k}=X_{i} X_{j} . . X_{k}$ and write differential equations for them using the constitutive equations for each of the $X_{i}$. The resulting equations are of the same type as Eq.(11). The similarity is obvious when one adopts a tensorial notation and defines products of the form $X^{(n)}=X \otimes \ldots \otimes X^{\dagger}$. The derivation of equations is specially simple within this notation.

In the computation of averages of quantities of physical interest, i.e. expected signal rates, appear also correlators of quantities at different times. In the most simple case

\footnotetext{
1 These results should be compared with the "cell" calculations presented in [15. In such approach the averaged probability is computed by direct "Monte-Carlo" methods. In order to simulate a finite correlation length a step function correlation is used: $f\left(t, t^{\prime}\right)$ is negligible whenever $\left|t-t^{\prime}\right|$ is greater than some characteristic scale, otherwise is a constant.
} 
expressions of the type $\left\langle X_{i}(t+\tau) X_{j}(t)\right\rangle$. We will show shortly that the knowledge of equal-time moments and the application of the quantum correlation theorem is sufficient for the computation of this kind of correlations.

The expected signal rate in a given experiment averaged over a finite range of energy and time can be described in general by a simplified expression of the form:

$$
N=\int_{\Delta E, \Delta t} d E d t \phi(E) P(E ; t)
$$

The function $\phi(E)$ is the experimental effective flux, where the original neutrino flux, the experimental detection and geometrical efficiencies and cross sections are included. A hidden dependence, potentially relevant, on $E_{0}, t_{0}$, the central points of the integration intervals, is understood.

For a fluctuating survival probability $P(E ; t), N$ is itself a random ,non Gaussian, variable. The averaged signal is simply the integral of the averaged probability. The second moment of the $\mathrm{N}$ distribution or more complicated energy or time correlations, i.e. averages of the type

$$
\left\langle N\left(E_{0}^{\prime}, t_{0}^{\prime}\right) N\left(E_{0}, t_{0}\right)\right\rangle
$$

are all of the same form, schematically:

$$
\left\langle N^{2}\right\rangle=\int_{\Delta E, \Delta t} d E_{1,2} d t_{1,2} \phi\left(E_{1}\right) \phi\left(E_{2}\right)\left\langle P\left(E_{1}, t_{1}\right) P\left(E_{2}, t_{2}\right)\right\rangle
$$

The correlation function appearing in the integrand of Eq. (33) can be computed in two steps. First we define the generalized density matrix $X^{(2)}$ as the tensorial product of usual density matrices at two different times and energies:

$$
X^{(2)}\left(E_{1}, E_{2} ; t_{1}, t_{2}\right) \equiv X\left(E_{1}, t_{1}\right) \otimes X\left(E_{2}, t_{2}\right) .
$$

The average of the element $\left\langle X_{1111}^{(2)}\right\rangle=\left\langle X_{11} X_{11}\right\rangle$ is evidently the probability correlation function we are looking for.

The differential equation for the equal time $X^{(2)}\left(E_{1}, E_{2} ; t, t\right)$ is obtained from the individual evolution equations for the matrices $X_{1,2} \equiv X\left(E_{1,2}\right)$ (indices $(1,2)$ label respectively expressions where $E_{1}, E_{2}$ appear)

$$
\partial_{t} X^{(2)} \equiv H_{1} X^{(2)}+X^{(2)} H_{2} \equiv \rho L X^{(2)}
$$

Eq.(35) is a random linear differential equation, linear in the stochastic variable $\rho(t)$. Applying the formalism developed in the previous section, we can write immediately the equation for the ensemble average $\left\langle X^{(2)}\right\rangle$ (Eq.(13-14)). Once we know the equal time correlator $\langle P(t) P(t)\rangle$, we obtain the expression for any other pair $\left(t, t^{\prime}\right)$ using the quantum regression theorem ( 16$]$ ) which reads as follows. For any vector Markov process $Y$, if the ensemble average of $Y$ fulfills a Schroedinger-like equation of the type

$$
\partial_{t}\langle Y(t)\rangle=G(t)\langle Y(t)\rangle
$$


with $G(t)$ an arbitrary matrix, then the second order correlations will obey the following equation

$$
\partial_{\tau}\left\langle Y_{i}(t+\tau) Y_{l}(t)\right\rangle=\sum_{j} G_{i j}(\tau)\left\langle Y_{j}(t+\tau) Y_{l}(t)\right\rangle
$$

Note that the quantum regression theorem is in principle not applicable to systems described by the integral equation Eq.(13). Nevertheless it is applicable to problems where the integral equation can be reduced to a ordinary differential equation as Eq.(31). Such second order equation can be easily expressed as a first order one by defining the auxiliary pair process $Y=\left(X, \partial_{t} X\right)$.

For the simplest case where $\rho$ is a $\delta$ correlated process the equation for non-equal time correlators is explicitly given by:

$$
\partial_{\tau}\left\langle X^{(2)}(t+\tau, t)\right\rangle=-i \Omega^{2} L^{2}(\tau)\left\langle X^{(2)}(t+\tau, t)\right\rangle .
$$

For exponentially correlated problems second order equations similar to Eq.(31) can be immediately obtained.

\section{Conclusions and final Remarks.}

In conclusion, in this work we have developed the general formalism for the study of neutrino propagation in stochastic media. This formalism has allowed us to derive systematically evolution equations for averaged quantities as survival probabilities and higher order moments of them. New integro-differential equations valid for finite correlation processes have been obtained for the first time. For exponentially correlated processes a second order ordinary equation is obtained as a consequence. The Redfield equation valid for Gaussian delta-correlated noise is rederived in a simple way as a particular case. In the context for neutrino oscillations, it has been shown that the presence of matter noise induces the appearance of a effective complex part in the matter density. The presence of magnetic field noise affects in a qualitatively different way. The wide range of validity of the $\delta$-correlated model has been checked in a realistic example corresponding neutrino oscillations in random solar matter. Finally, the formalism together with the quantum correlation theorem has been used to obtain equations for higher distribution moments and non-equal time correlation functions.

The formalism can be generalized in an obvious way to perform the ensemble average of equations of slightly more general type than Eq.(11), equations of the form:

$$
i \partial_{t} X=\sum_{i} \rho_{i}(t) L_{i}(t) X ; \quad X(0)=X_{0}
$$

These equations appear for example in chaotic neutrino magnetic precession. The result for the case where the $\rho_{i}$ are $\delta$-correlated in time but mutually uncorrelated is simply:

$$
i \partial_{t}\langle X(t)\rangle=-i \sum_{i} \Omega_{i}^{2} L_{i}^{2}(t)\langle X(t)\rangle .
$$


The generalization to continuous Schroedinger equations in presence of random potentials or random external forces is also obvious if we left aside the mathematical differences coming from the appearance of infinite dimensional Hilbert spaces. The formalism is of general application to any quantum system governed by similar Schroedinger equations: equations where a stochastic function appears multiplicatively in some term of the Hamiltonian.

\section{Acknowledgments.}

This work has been supported by DGICYT under Grant PB95-1077 and by a DGICYTMEC contract at Univ. de Valencia. Early versions of this work were developed at the Institute Fur Theoretische Physik, Universitat Bern, supported by a grant from the Wolferman-Nageli Foundation. 


\section{References}

[1] A. Nicoladis. Phys. Lett. 262 (1991) 2,3, pp. 303-306.

[2] F.N. Loreti, A.B. Balantekin. Phys. Rev. D50 (1994), pp. 4762-4770.

[3] K. Enqvist, V. Semikoz. Phys. Lett. B312, 310 (1993).

[4] F.N. Loreti, YZ Qian, G.M. Fuller, A.B. Balantekin. astro-ph/9508106.

[5] E. Torrente-Lujan. hep-ph/9602398.

[6] H. Nunokawa, A. Rossi, V.B. Semikoz, J.W.F. Valle. IFIC/95-49, hep-ph/9602307.

[7] C.P. Burgess, D. Michaud. hep-ph/9606295.

[8] P. Bamert, C.P. Burgess, D. Michaud. hep-ph/9707542.

[9] V.B. Semikoz. Phys. Rev. D48, N.11 (1993) 5264-5273.

[10] K. Enqvist, A.I. Rez, V.B. Semikoz. Nucl. Phys. B436, (1995) 49-64.

[11] J. Zinn-Justin, Quantum Field Theory and critical phenomena., (International Series of monographs on Physics.N-77. Oxford Science Publications. 1990.)

[12] E. Torrente-Lujan, hep-ph/9807371.

[13] E. Torrente-Lujan, hep-ph/9807426.

[14] V.B. Semikoz, E. Torrente-Lujan. Work in preparation.

[15] C.P. Burgess. hep-ph/9711425

[16] G.W. Gardiner, Handbook of stochastic methods. ( Springer Verlag, 1975). 

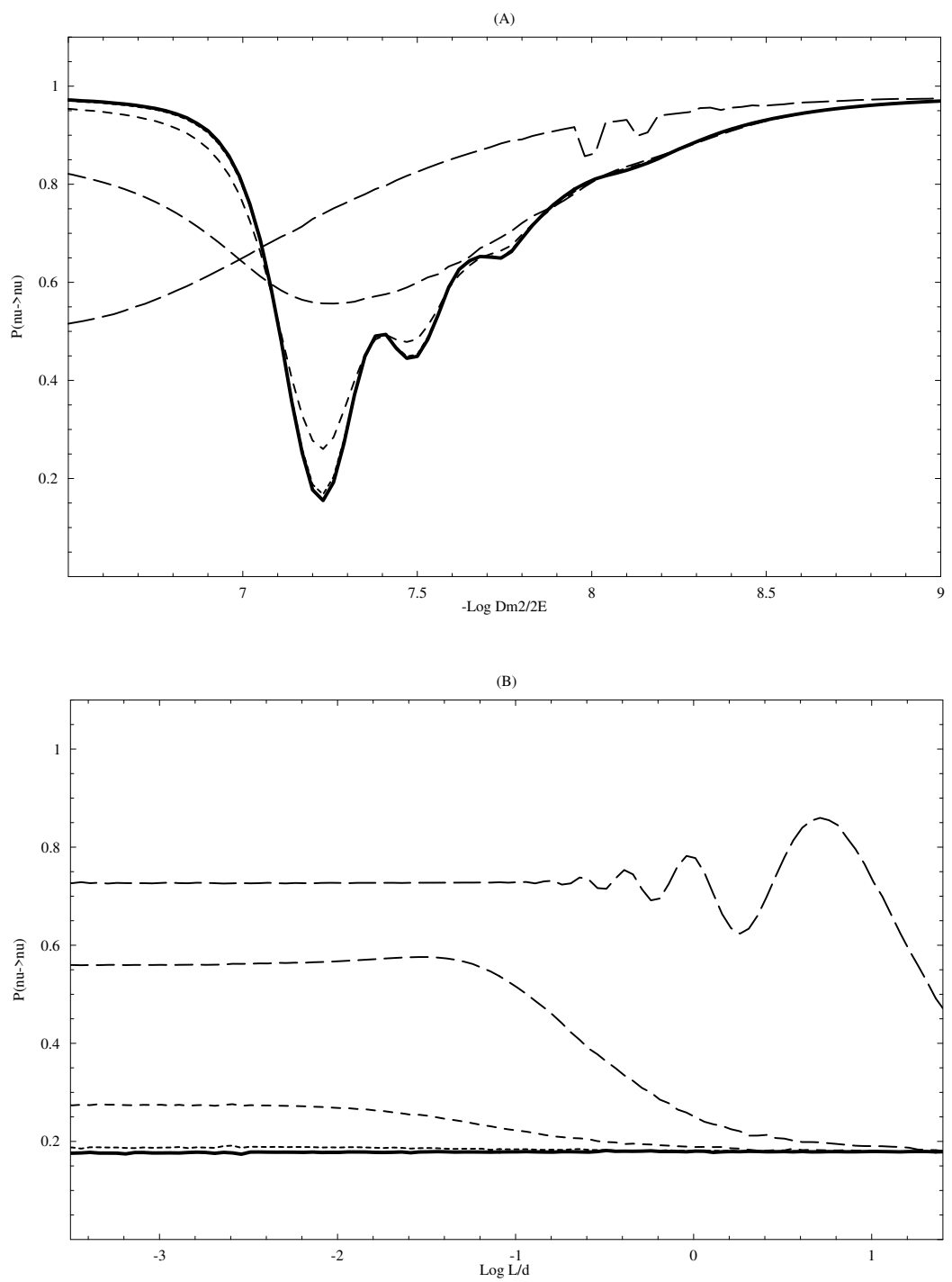

Figure 1: Neutrino oscillations in presence of random solar matter. (A) Averaged survival probability as a function of $\Delta m^{2} / 2 E$ ( $\delta$-correlation case, Eq.(14 )). Continuos curve: absence of noise. Dashed lines; $\Omega^{2}=100,1,0.1,0.01(\times 1000 \mathrm{Km}$, from longer to shorter dashing). (B) The neutrino survival probability is plotted as a function of $L / d$ with $L \equiv 1 / \epsilon, " \mathrm{~d}$ " is the distance over which the chaotic field is acting $\left(\approx 0.6 R_{\odot}\right)$. Eq.(31) is used for the same values of $\Omega^{2}$ as before $\left(\Delta m^{2} / 2 E=6.310^{-8} \mathrm{eV}^{2} / \mathrm{MeV}\right.$ now $)$. For both plots, the neutrino, created at $r \approx 0.4 R_{\odot}$ as a pure electron neutrino, propagates radially outwards. The flavour mixing angle is $\sin ^{2} 2 \theta=0.05$. 